\title{
The $g_{2}$ Spin Structure Function
}

\section{Melissa Cummings*}

The College of William and Mary

E-mail: melissacajlab.org

Jefferson Lab has been at the forefront of a program to study the polarized structure of nucleons. Measurements of the spin-dependent structure functions, $g_{1}$ and $g_{2}$, have proven to be powerful tools in testing and understanding Quantum Chromodynamics. To measure $g_{2}$ a transversely polarized target is needed, which proves to be challenging experimentally. Prior to JLab, the only dedicated experiment to measure $g_{2}$ was SLAC E155x, which shows consistency with the leading twist $g_{2}^{W W}$ prediction, but has large uncertainties. The function $g_{2}^{n}$ has been measured extensively in Hall A at JLab over a wide range of $Q^{2}$. The Resonances Spin Structure (RSS) experiment in Hall $\mathrm{C}$ gave a precision measurement of $g_{2}$ for the proton and deuteron at intermediate $Q^{2}$, providing the first world data for $g_{2}^{p, d}$ in the nucleon resonance region. The Spin Asymmetries of the Nucleon experiment (SANE), also performed in Hall C, provided a measurement of $g_{2}$ in the high $Q^{2}$ (DIS) region. More recently, the $g_{2}^{p}$ experiment took data covering the low $Q^{2}$ region. The $0^{\text {th }}$ moment of $g_{2}$ provides a test of the Burkhardt-Cottingham sum rule, which states that the integral of $g_{2}$ over the Bjorken scaling variable $\mathrm{x}$ goes to zero. This sum rule, valid for all values of $Q^{2}$, has been satisfied for the neutron, but a violation is suggested for the proton at high $Q^{2}$. The $2^{\text {nd }}$ moment allows for a benchmark test of lattice QCD predictions at high $Q^{2}$ with $d_{2}$, and a test of Chiral Pertubation Theory at low $Q^{2}$. Specifically, the behavior of the longitudinallytransverse spin polarizability $\left(\delta_{L T}\right)$, as $\chi \mathrm{PT}$ calculations of this quantity deviate significantly from the measured neutron data. This document will provide an overview of the JLab effort to measure $g_{2}$, with a focus on the more recent $g_{2}^{p}$ experiment; the current status of the analysis will be discussed along with preliminary results.

XXII. International Workshop on Deep-Inelastic Scattering and Related Subjects,

28 April - 2 May 2014

Warsaw, Poland

* Speaker. 


\section{Introduction and Motivation}

The proton's composition of quarks and gluons, which exhibit many-body interactions, make scattering from a proton significantly more complicated than a point-like particle. The four structure functions, $F_{1}, F_{2}, g_{1}$ and $g_{2}$ describe the deviation of a nucleon from point-like behavior in an inclusive scattering experiment, $N\left(e, e^{-}\right)$. For the case with an unpolarized nucleon and unpolarized electron, the scattering cross section can be described with the functions $F_{1}$ and $F_{2}$ (equation 1.1). For the more complicated case, with a polarized nucleon and polarized electron, two additional structure functions ( $g_{1}$ and $g_{2}$ ) are necessary to completely parameterize the scattering cross section (equations 1.2 and 1.3). We can investigate the spin structure of the nucleon by using different combinations of the beam/target polarization, i.e. longitudinally polarized electrons with a longitudinally or transversely polarized nucleon.

$$
\begin{gathered}
\frac{d^{2} \sigma}{d \Omega d E^{\prime}}=\sigma_{M o t t}\left[\frac{1}{v} F_{2}\left(x, Q^{2}\right)+\frac{2}{M} F_{1}\left(x, Q^{2}\right) \tan ^{2}(\theta / 2)\right] \\
\Delta \sigma_{\|}=\frac{d^{2} \sigma}{d \Omega d E^{\prime}}(\downarrow \Uparrow-\uparrow \Uparrow)=\frac{4 \alpha^{2}}{M Q^{2}} \frac{E^{\prime}}{v E}\left[\left(E+E^{\prime} \cos \theta\right) g_{1}\left(x, Q^{2}\right)-\frac{Q^{2}}{v} g_{2}\left(x, Q^{2}\right)\right] \\
\Delta \sigma_{\perp}=\frac{d^{2} \sigma}{d \Omega d E^{\prime}}(\downarrow \Rightarrow-\uparrow \Rightarrow)=\frac{4 \alpha^{2} \sin \theta}{M Q^{2}} \frac{E^{\prime 2}}{v^{2} E}\left[v g_{1}\left(x, Q^{2}\right)-2 E g_{2}\left(x, Q^{2}\right)\right]
\end{gathered}
$$

where $v$ is the difference between the incoming and scattered electron energy, $Q^{2}$ is the 4momentum transfer squared, and $x$ is the Bjorken scaling variable.

To interpret these structure functions, we can start with the Quark-Parton model, which was put forth by Feynman in 1969. In the Bjorken scaling limit, the structure functions $F_{1}, F_{2}$ and $g_{1}$ can be written in terms of parton distribution functions. However, as this model describes the partons as point-like non-interacting particles, $g_{2}$ has a value of zero. This is where we begin to see the cracks in the so-called "naive" quark model, as $g_{2}$ contains contributions from quark-gluon interactions. The function $g_{2}$ is usually expressed in terms of its twist expansion.

$$
g_{2}\left(x, Q^{2}\right)=g_{2}^{W W}\left(x, Q^{2}\right)+\bar{g}_{2}\left(x, Q^{2}\right)
$$

The first term of equation $1.4, g_{2}^{W W}$, is known as the Wandzura-Wikczek relation; the leading twist-2 term which expresses $g_{2}$ entirely in terms of $g_{1}$. The second term, $\bar{g}_{2}$, contains terms that are twist-3, which arise from quark-gluon interactions. Higher twist processes can be thought of as involving more than one parton of the hadron in the scattering process. Instead of viewing only a bare quark, we begin to probe how the quarks and gluons interact in the context of the nucleon. The function $g_{2}$ offers the most direct view of these correlations, so it becomes an attractive quantity to measure.

\section{Measurements of the $g_{2}$ Structure Function at Jefferson Lab}

Measurements of the $g_{2}$ structure function require a target which is polarized transversely with respect to the polarization of the electron beam, which is more difficult experimentally. Prior to 


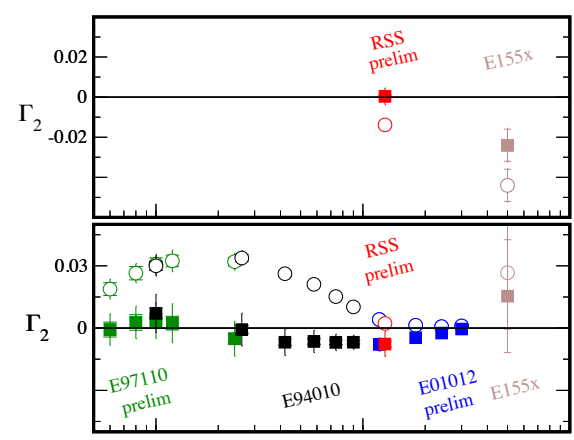

Figure 1: Current results for the BC sum rule. The top plot is for the proton and the bottom plot is for the neutron. Open circles represent the measured data, while black diamonds show the total integral with low-X and elastic contributions included. Reproduced from [8].

measurements at JLab, the only dedicated experiment to measure $g_{2}$ was SLAC E155x [6], which shows consistency with the $g_{2}^{W W}$ prediction. The function $g_{2}^{n}$ has been measured extensively in Hall A at JLab [8], but data on $g_{2}^{p}$ remain scarce. The Resonances Spin Structure (RSS) experiment in Hall C gave a precision measurement of $g_{2}$ for the proton and deuteron at intermediate $Q^{2}$, providing data for $g_{2}^{p, d}$ in the nucleon resonance region [1]. The Spin Asymmetries of the Nucleon experiment (SANE), also performed in Hall C, provided a measurement of $g_{2}$ in the high $Q^{2}$ (DIS) region [7]. More recently, the $g_{2}^{p}$ experiment took data covering the low $Q^{2}$ region.

The $0^{\text {th }}$ moment of $g_{2}$ (no $x$-weighting) provides a test of the Burkhardt-Cottingham sum rule, shown in equation 2.1. The BC sum rule is not a parton model prediction, and is valid for all values of $Q^{2}$. Current results for tests of the BC sum rule are show in figure 1. For the neutron, the BC sum rule has been tested over a large range of $Q^{2}$ and show consistency with the expected result of zero, within small uncertainties. The lack of data for the proton leaves the $\mathrm{BC}$ sum rule largely untested. The results from SLAC suggest an inconsistency on the level of $2.75 \sigma$, which arrises from both a large experimental uncertainty as well as uncertainty associated with the low-x extrapolation that is difficult to quantify. The results from the RSS experiment show better agreement; the integral over the measured region is negative, but is consistent with zero once the low-x extrapolation is added in. Precision data from JLab is consistent with the BC sum rule in all cases, which suggests that $g_{2}$ is a well behaved function with good convergence. The data from the $g_{2}^{p}$ experiment will provide an additional test for the proton at low $Q^{2}$.

$$
\Gamma_{2}=\int_{0}^{1} g_{2}\left(x, Q^{2}\right) d x=0
$$

The $2^{\text {nd }}$ moment of $g_{2}$ provides insight o the generalized spin polarizabilities $\gamma_{0}$ and $\delta_{L T}$ (equations 2.2 and 2.3), which offer a benchmark test of Chiral Perturbation Theory ( $\chi$ PT). For the quantity $\gamma_{0}$, the difficulty in these calculations is in how to included the resonances, specifically the $\Delta$ resonance, which usually dominates.

$$
\gamma_{0}\left(Q^{2}\right)=\frac{16 \alpha M^{2}}{Q^{6}} \int_{0}^{x_{0}} x^{2}\left[g_{1}\left(x, Q^{2}\right)-\frac{4 M^{2}}{Q^{2}} x^{2} g_{2}\left(x, Q^{2}\right)\right]
$$



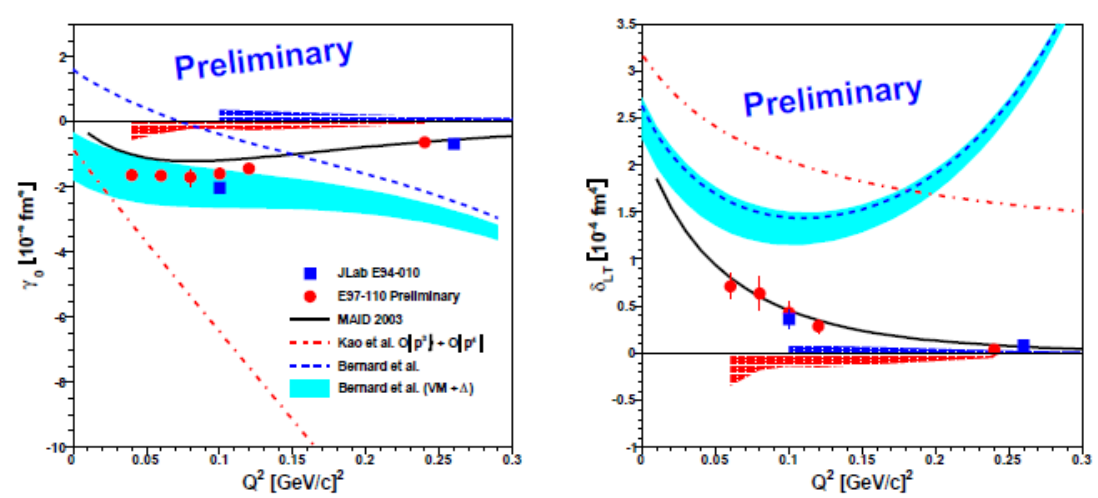

Figure 2: On the left are the current neutron results for the generalized spin polarizability $\gamma_{0}$, and on the right are neutron results for $\delta_{L T}$. The blue squares represent published data from E94010, while the red circles are preliminary data from E97110. Reproduced from [9].

$$
\delta_{L T}\left(Q^{2}\right)=\frac{16 \alpha M^{2}}{Q^{6}} \int_{0}^{x_{0}} x^{2}\left[g_{1}\left(x, Q^{2}\right)+g_{2}\left(x, Q^{2}\right)\right]
$$

The neutron results for the generalized polarizabilites are shown in figure 2. Shown alongside the data for $\gamma_{0}$ is the relativistic baryon $\chi$ PT prediction (represented by the blue dotted line) and the same calculation, but explicitly including the $\Delta$ resonance and vector meson contributions (light blue band). The prediction at low $Q^{2}$ matches well with the experimental results, but there is disagreement at high $Q^{2}$. The quantity $\delta_{L T}$ is thought to be a better testing ground for $\chi \mathrm{PT}$ due to its insensitivity to the $\Delta$ resonance. In this case, as seen in figure 2, explicitly including the vector meson and $\Delta$ contribution shifts the central value of the prediction, but does not change the overall shape, indicating that the $\Delta$ contribution is not large for these calculations. However, there is clearly a large difference between the data and predictions for $\delta_{L T}$ [2]. The $g_{2}^{p}$ experiment will provide an additional test of these quantities at low $Q^{2}$ for the proton.

\section{The Experiment}

The $g_{2}^{p}$ experiment ran from March to May of 2012. An inclusive measurement was performed in the low $Q^{2}$ region $0.02<Q^{2}<0.2 \mathrm{GeV}^{2}$ at forward angles to obtain the proton spin-dependent cross sections. To extract $g_{2}$, both $\Delta \sigma_{\|}$and $\Delta \sigma_{\perp}$ are needed. This experiment measured $\Delta \sigma_{\perp}$, results from the Hall B EG4 experiment will be used as input for $\Delta \sigma_{\|}$. Some data were taken in the longitudinal setting during this experiment as a cross check of the Hall $\mathrm{B}$ results. The kinematic coverage achieved during the experiment is shown in figure 3.

This experiment required a large scale installation in Hall A (see figure 4). A solid ammonia target was polarized through the process of dynamic nuclear polarization. In order to compensate for the deflection of the beam by the large target magnetic field, a pair of chicane magnets were installed upstream of the target. To reach the small scattering angle of $5.69^{\circ}$ necessary for this kinematic range, a septum magnet was installed downstream of the target. New beamline diagnostics, to monitor the beam position and beam current, were required due to the low beam current 


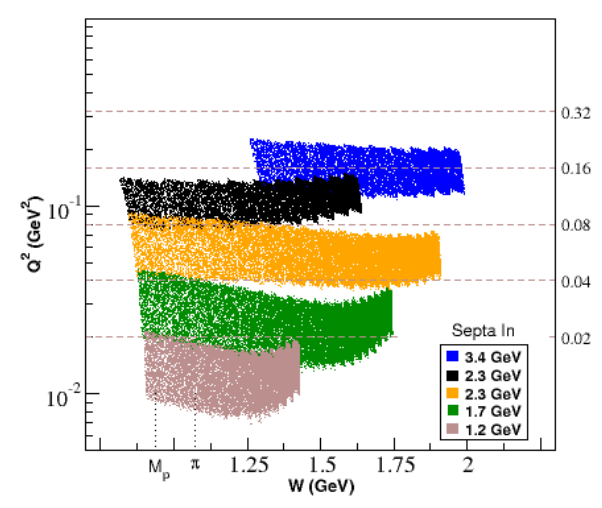

Figure 3: Kinematic coverage of the $g_{2}^{p}$ experiment.

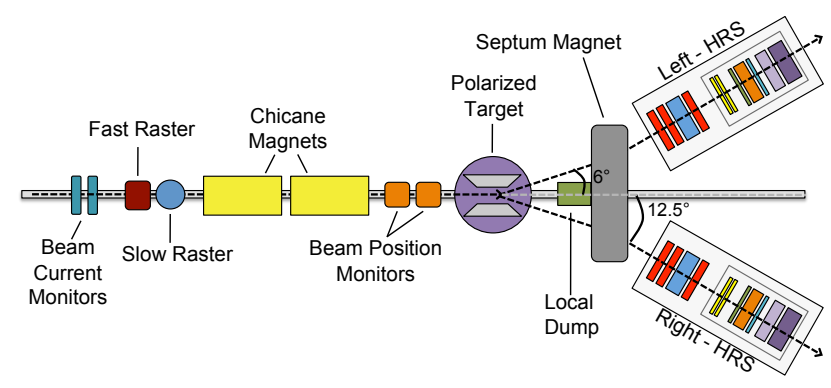

Figure 4: Hall A beamline for the $g_{2}^{p}$ experiment.

(50-100 nA) needed to maintain the target polarization. For certain kinematics, a local beam dump was necessary, located just downstream of the septum magnet. The standard Hall A high resolution spectrometers (HRS) were used with a detector stack containing two pairs of vertical drift chambers (VDC), two planes of scintillators, a gas Cherenkov counter, and a lead glass calorimeter. The VDCs provide tracking information for the scattered electrons. The two planes of scintillators, placed $\sim 2 \mathrm{~m}$ apart, provide the trigger for the data acquisition system. Sandwiched between the scintillator planes is a gas Cherenkov counter, which contains $\mathrm{CO}_{2}$. At these kinematics, electrons that pass through the gas will produce Cherenkov light, whereas heavier particles will not. Finally, two layers of lead glass form an electromagnetic calorimeter; energetic particles that pass through the material will produce a cascade of secondary particles. As this "shower" of particles propagates through the lead glass, the particle's energy is converted to light, which is collected and used to determine the initial energy of the particle. The gas Cherenkov and lead glass calorimeter are used for particle identification.

\section{Status of Analysis}

Analysis of the $g_{2}^{p}$ data is pushing forward. Detector calibration and efficiency studies have been completed for the gas Cherenkov, lead glass calorimeters and scintillator detectors in addition 

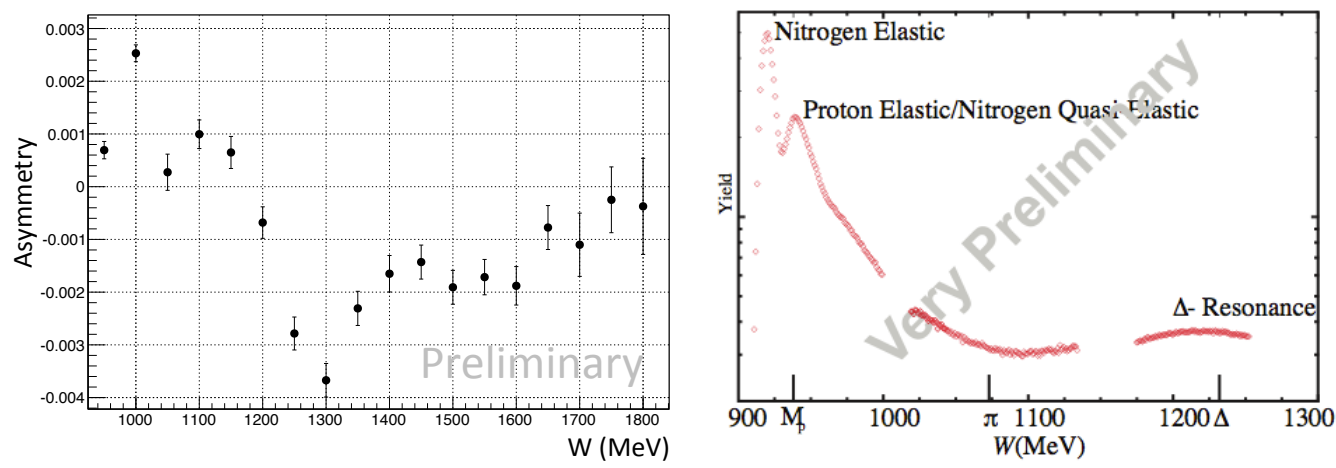

Figure 5: Preliminary reults for the $g_{2}^{p}$ experiment. On the left are asymmetry results (normalized by beam and target polarizations) and on the right are yield results.

to tracking analysis, including analysis of multi-track events. Scaler analysis, including beam current monitor calibrations, helicity decoding and deadtime calculations are finished. The target polarization analysis is also completed, with an average polarization of $\sim 70 \%$ and $\sim 15 \%$ for the 5T and 2.5T settings, respectively. Optics analysis was made more difficult for this experiment with the large target magnetic field, the septum field, and chicane magnets, but a first round of optics calibrations for the left HRS with target field on have been finished. Beam position calibrations were recently completed, and raster size calibrations are currently underway. Extraction of the packing fraction and dilution factor, necessary to understand the fraction of events that scattered from unpolarized target material, is in progress. In addition, analysis of elastic events and radiative corrections for cross section analysis are currently underway. Preliminary asymmetries and yields can be seen in figure 5 . The asymmetry has been scaled by the beam and target polarization, but does not include the dilution factor. The uncertainty show here is purely statistical, and radiative corrections have not been included. In the yields, we see good separation between the nitrogen and hydrogen elastic peaks. Additional data was taken on carbon, empty target cells, and helium to be used in dilution factor analysis. We expect to have preliminary results for $g_{2}$ in $\sim 6-12$ months.

\section{Summary}

In these proceedings, we have discussed the measurements of the $g_{2}$ structure function for the proton. From Jefferson Lab, the RSS and SANE experiments have provided measurements of this spin observable at intermediate and high $Q^{2}$, but the most recent $g_{2}^{p}$ experiment will provide the first measurement of this quantity at low $Q^{2}$. These data will provide a benchmark test for $\chi \mathrm{PT}$ calculations of the generalized spin polarizabilities $\gamma_{0}$ and $\delta_{L T}$. Previous tests for the neutron suggest a discrepancy between data and calculations; these data will provide further insight in the low $Q^{2}$. In addition, they will allow for a test of the Burkhardt-Cottingham sum rule at low $Q^{2}$ for the proton. This sum rule is satisfied for the neutron, but lack of data for $g_{2}^{p}$ leaves it largely untested for the proton. 


\section{References}

[1] F. R. Wesselmann et al. [RSS Collaboration], Phys. Rev. Lett. 98, 132003 (2007).

[2] M. Amarian et al. [Jefferson Lab E94010 Collaboration], Phys. Rev. Lett. 93, 152301 (2004).

[3] V. Bernard, T. R. Hemmert and U. G. Meissner, Phys. Lett. B 545, 105 (2002).

[4] C. W. Kao, T. Spitzenberg and M. Vanderhaeghen, Phys. Rev. D 67, 016001 (2003).

[5] V. Bernard, E. Epelbaum, H. Krebs and U. G. Meissner, arXiv:1209.2523v1 [hep-ph] (2012).

[6] P.L. Anthony et al. [SLAC E155 Collaboration] Phys. Lett. B 553, 18 (2003)

[7] S. Choi,M. Jones,Z. E. Meziani, O. Rondon-Aramayo Experimental Proposal: "Spin Asymmetries of the Nucleon Experiment" (2006)

[8] J.-P. Chen, arXiv:1001.3898v1 [nucl-ex] (2010).

[9] V. Sulkosky, PoS CD12 (2013) 023. 